\title{
Effect of thermal exposure on the tensile properties of Kevlar fibres
}

\author{
H. V. PARIMALA, K. VIJAYAN \\ Materials Science Division, National Aeronautical Laboratory, Bangalore 560 017, India
}

Kevlar, belonging to the family of high-strength, high-modulus aramid fibres, has an extensive range of applications [1]. It is likely that while in use, the fibres will be exposed either intentionally or accidentally to thermal environments, which in turn can alter their properties and performance. In this letter, we report some observations concerning the effect of cumulative thermal exposure on the tensile properties of Kevlar fibre. It must be mentioned that Hindeleh and Abdo [2,3] have already studied the effect of thermal exposures on Kevlar fibres under very specific conditions, namely exposures in an atmosphere of nitrogen gas for a duration of $15 \mathrm{~min}$ at temperatures above $150^{\circ} \mathrm{C}$. This study considered the effects of thermal exposures that bear a closer resemblance to what the fibres would experience in their service life, i.e. exposures in an uncontrolled atmosphere of air and for durations significantly longer than $15 \mathrm{~min}$.

The samples used were Kevlar 49 fibres. Unconstrained bundles of the fibres were heat-treated in a tubular resistance furnace in which the temperature could be controlled and maintained stable within $\pm 5^{\circ} \mathrm{C}$. The temperatures $(T)$ chosen for exposing the fibres were 150,250 and $350^{\circ} \mathrm{C}$. The duration $(t)$ of the cumulative exposure varied between 0.5 and $260 \mathrm{~h}$. The values of $t$ corresponding to 250 and $350{ }^{\circ} \mathrm{C}$ were, however, limited by the thermally induced brittleness of the fibre. It was found that after 250 and $6 \mathrm{~h}$ exposure to 250 and $350{ }^{\circ} \mathrm{C}$, respectively, the fibres turned brittle and crumbled with handling. They could not therefore be used further for tensile testing. In contrast, fibres exposed to $150{ }^{\circ} \mathrm{C}$ could be handled comfortably even after $260 \mathrm{~h}$ exposure. These observations, although qualitative, suggest that exposures to 350 and $250^{\circ} \mathrm{C}$ reduce the tensile strength of the fibre. The quantitative data presented below substantiate this view. The tensile properties of single filaments were analysed on a Zwick universal testing machine. The filaments were pulled in tension, at a rate of $2.5 \mathrm{~mm} \mathrm{~min}^{-1}$ and the chart recording was carried out at a rate of $60 \mathrm{~mm} \mathrm{~min}^{-1}$. Each of the values reported in this letter is an average obtained by examining at least 50 specimens.

Fig. 1 compares the typical stress-strain curves reported from filaments both before and after the thermal exposures. The striking features of these curves are, first, the progressive reduction in the breaking stress and strain with thermal exposure, and secondly, the nearly invariant slope. Fig. 2 shows the variation in the fractional values of the tensile strength $(S)$, tensile modulus $(M)$ and

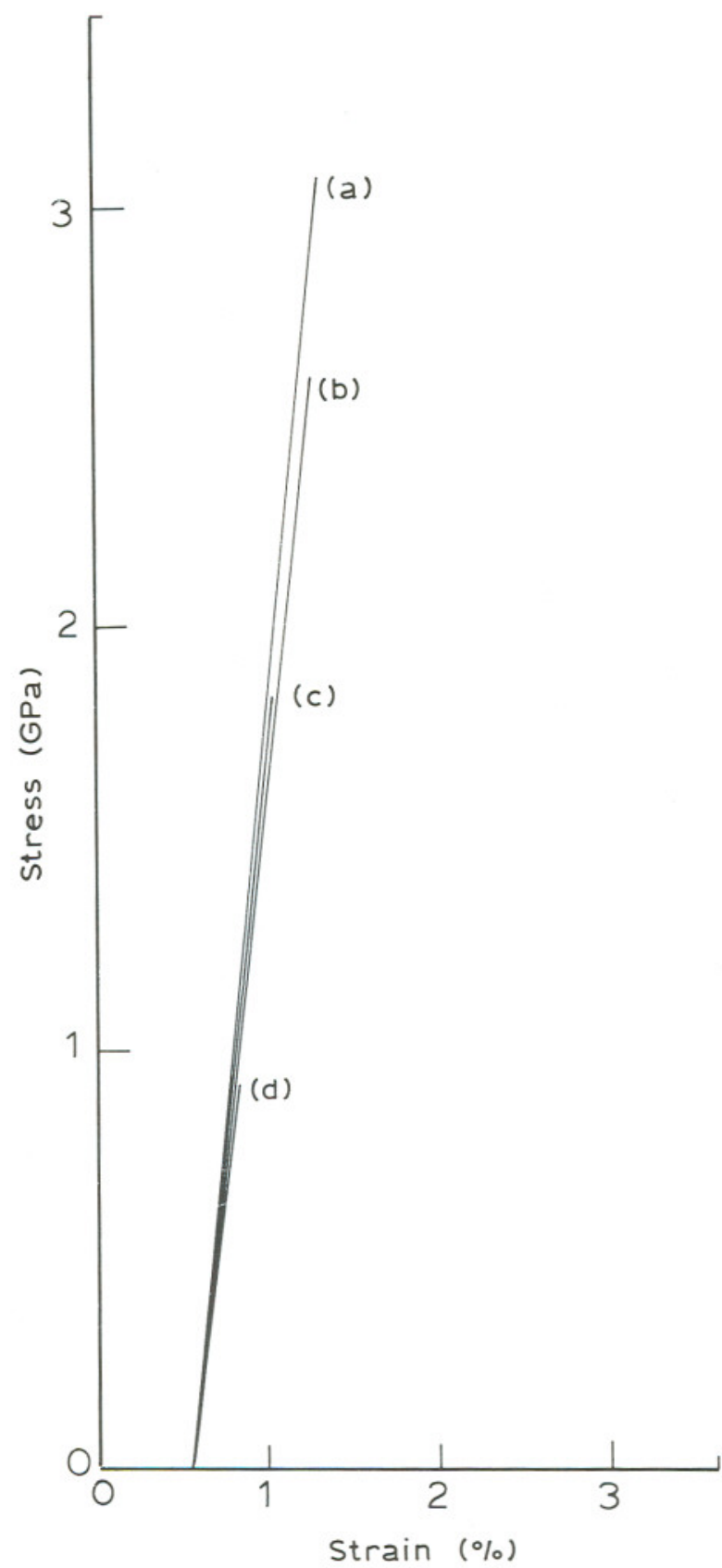

Figure 1 Typical stress-strain curves for Kevlar filaments (a) before, and after thermal exposure at (b) $150{ }^{\circ} \mathrm{C}$, (c) $250^{\circ} \mathrm{C}$ and (d) $350{ }^{\circ} \mathrm{C}$.

percentage elongation at break $(\varepsilon)$. Here $S(t), M(t)$ and $\varepsilon(t)$ represent the respective values of the tensile strength, tensile modulus and percentage elongation at break for fibres exposed to temperature $T$ for duration $t$, and $S(0), M(0)$ and $\varepsilon(0)$ represent the corresponding values before subjecting the fibres to thermal exposure. For the samples used 

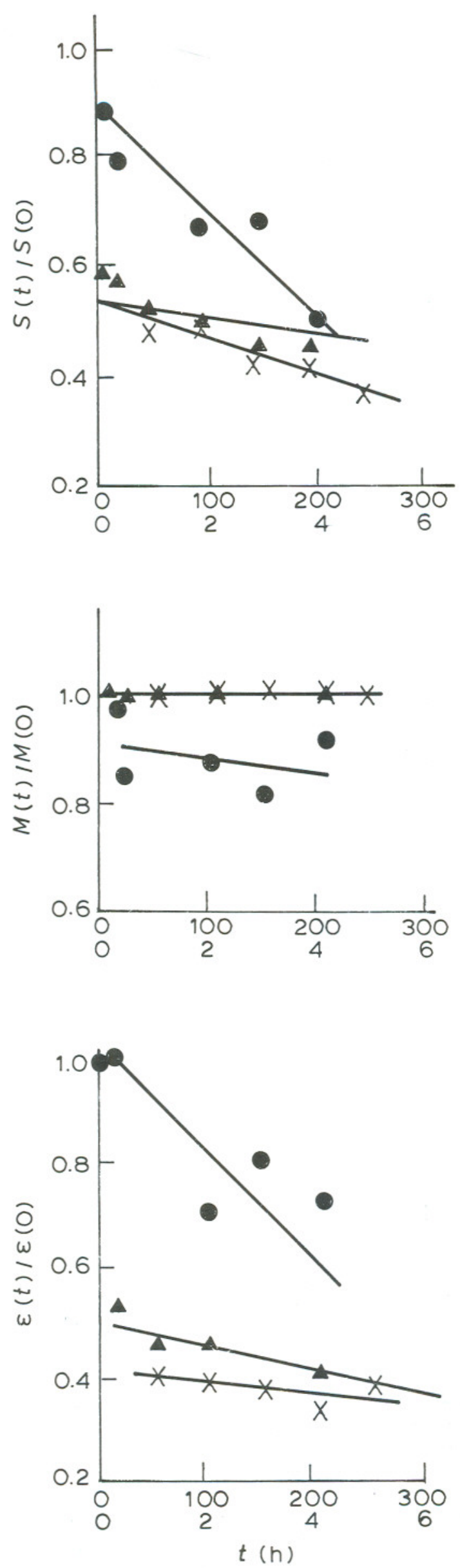

Figure 2 Variation in the fractional values of the tensile strength $(S)$, tensile modulus $(M)$ and percentage elongation at break $(\varepsilon)$ with thermal exposure at $150^{\circ} \mathrm{C},(\boldsymbol{\Delta}) 250^{\circ} \mathrm{C}$ and $(\times) 350^{\circ} \mathrm{C}$. The lower scale of $0-6 \mathrm{~h}$ on the $t$-axis is relevant only for $350^{\circ} \mathrm{C}$. in this study the values of $S(0), M(0)$ and $\varepsilon(0)$ averaged $2.8 \pm 0.4 \mathrm{GPa}, 100 \pm 8 \mathrm{GPa}$ and $3.0 \pm 0.4$, respectively. It may be pointed out that, although the estimated standard deviations quoted here appear to be high, estimated standard deviations of this order are generally accepted [4] and reflect the scatter commonly observed for these fibres.

Fig. 2 shows that the first exposures to 350,250 and $150{ }^{\circ} \mathrm{C}$ for the specified durations reduce the tensile strength of the fibre by approximately 50,40 and $10 \%$, respectively. The rate at which further reduction progresses with $t$ is, however, highest for the filaments exposed to $150{ }^{\circ} \mathrm{C}$. Fig. 2 also shows that with about $3 \mathrm{~h}$ exposure to $350{ }^{\circ} \mathrm{C}$ the tensile strength is reduced by about $60 \%$. Reduction by nearly the same extent is found to occur at $150{ }^{\circ} \mathrm{C}$ itself when the exposure time is increased to about $250 \mathrm{~h}$. This feature clearly shows that the thermally induced effects are controlled by both the temperature and the duration of the exposure. The changes induced at any temperature $T_{2}$ can occur at a temperature $T_{1}\left(<T_{2}\right)$ if the exposure to $T_{1}$ is sufficiently long. It is therefore essential that when empirical equations are used to describe the thermally induced effects, they should include both the variables $T$ and $t$.

The nearly identical slopes of the stress-strain curves in Fig. 1 suggest that the tensile modulus is only slightly affected by thermal exposures. Fig. 2 shows that exposures to 250 and $350{ }^{\circ} \mathrm{C}$ do not introduce any significant reduction in the tensile modulus. However, with exposures to $150{ }^{\circ} \mathrm{C}$ the tensile modulus tends to reduce by approximately $10 \%$. These features suggest that exposures to 250 and $350{ }^{\circ} \mathrm{C}$ may introduce some structural changes that enable retention of the tensile modulus. At $150{ }^{\circ} \mathrm{C}$ these changes either have not commenced or are not complete. It must be mentioned that X-ray diffraction patterns recorded from heat-treated fibres do indicate the occurrence of structural changes (possibly recrystallization) in fibres exposed to 250 and $350^{\circ} \mathrm{C}$ [5]. Hindeleh and Abdo [2] also reported an increase in the tensile modulus induced by approximately $15 \mathrm{~min}$ exposure to $400^{\circ} \mathrm{C}$ and correlated it with the increase in the crystallinity of the fibre. Fig. 2 shows that the variation in the percentage elongation at break is very similar to the variation in tensile strength.

The above data suggest that with thermal exposures, the tensile strength of Kevlar fibres deteriorates more easily than the tensile modulus. It is therefore likely that the structural characteristics responsible for the high tensile strength of the fibre are preferentially affected by the thermal exposures. It is well known [6] that the high tensile strength of Kevlar fibres is closely connected with its specific crystal structural characteristics, which enable efficient transfer of load. In particular, the regular network of hydrogen bonds in each layer, i.e. the crystallographic $b c$-plane, facilitates the transfer of load between adjacent polymer chains and the van der Waals forces present along the crystallographic $a$-direction facilitate the transfer of load between 
adjacent layers. Disruption of either or both of these load transfer mechanisms can result in a reduction in the tensile strength of the fibre. In the crystal structure of Kevlar fibres, the unit-cell length $b$ represents the interchain distance and is hence connected with the scheme of hydrogen bonds which link adjacent chains. In a similar manner, the value of $a / 2$, which represents the interlayer distance, is related to the van der Waals interactions. Any disruption in the scheme of hydrogen bonds and the van der Waals interactions could be expected to manifest as corresponding changes in the $b$ - and the $a$-length, respectively. Our X-ray data [7] have shown that the effect of thermal exposure on the unit-cell constants is to introduce a preferential increase in the $a$-value, which in turn indicates that the interlayer interactions are weakened by thermal exposures. Conclusive X-ray evidence for thermally induced changes in the $b$-value has not been obtained by us. It is, however, noted that the increase in the $a$-value (approximately $2 \%$ for $T=350{ }^{\circ} \mathrm{C}$ and $t=5 \mathrm{~h}$ ) is not commensurate with the reduction in tensile strength shown in Fig. 2. It is therefore likely that in addition to the weakening of the interlayer interactions, there are other factors that also contribute to the deteriorations in the tensile strength.

Using the data presented in Fig. 2, the activation energy $(E)$ required to reduce the tensile strength by $50 \%$ was calculated from

$$
t_{1 / 2}=A \exp (-E / R T)
$$

where $t_{1 / 2}$ is the time (in $\mathrm{min}$ ) required for a $50 \%$ reduction at temperature $T$ (in $\mathrm{K}$ ), $R$ is the gas constant and $A$ is the pre-exponential factor. From the slope of the $\ln t_{1 / 2}$ versus $1 / T$ curve, the value of $E$ was estimated to be $54 \mathrm{~kJ} \mathrm{~mol}^{-1}$. This value is higher than that required to disrupt a single hydrogen bond and less than that needed to disrupt a covalent bond along the chain [8]. The low value of $E$ provides further evidence that even partial disruption of the van der Waals forces and/or the hydrogen bonds in the crystal structure is sufficient to introduce a $50 \%$ reduction in the tensile strength of the fibre.

In the course of the study on the tensile proper- ties, it was also noted that thermal exposure of Kevlar fibres was accompanied by visually detectable changes in the colour of the fibre. After $2 \mathrm{~h}$ exposure to $350{ }^{\circ} \mathrm{C}$ and $20 \mathrm{~h}$ exposure to $250^{\circ} \mathrm{C}$ the fibres turned brownish and the degree of brownness increased with a further increase in $t$ as well as $T$. Fibres exposed to $150{ }^{\circ} \mathrm{C}$ retained their initial yellowish lustre even after $250 \mathrm{~h}$ exposure. Thus, the colour of the fibre may itself provide useful evidence for any accidental exposure that might have occurred for the fibre.

\section{Acknowledgements}

The authors thank the Aeronautical Research and Development Board of India for sanctioning a project under which this work was carried out. The authors sincerely thank $\mathrm{Mr}$ M. K. Sridhar for providing the sample used in this study. They are very grateful to Dr P. S. Gopalakrishnan and $\mathrm{Mr}$ P. $\mathrm{S}$. Lakshminarasimham for the assistance given in heating the samples. They sincerely acknowledge Dr N. Balasubramanian and Mr Basavaraj of Everest Building Products, Bangalore, for enabling the tensile tests to be performed. They are also thankful to Dr A. K. Singh for the support.

\section{References}

1. S. W. KWOLEK, W. MEMEGER and E. V. TRUMP, in "Polymers for advanced technologies", edited by M. Lewin (VCH Publishers, FRG, 1988) p. 450.

2. A. M. HiNdELEH and SH. M. ABDO, Polymer 30 (1989) 218.

3. Idem, Polym. Commun. 30 (1989) 184.

4. M. G. DOBB and R. M. ROBSON, J. Mater. Sci. 25 (1990) 459.

5. H. V. PARIMALA, MPhil dissertation, Mangalore University (1991).

6. M. G. MORTHOLT, in "Recent advances in liquid crystalline polymers", edited by L. L. Chapoy (Elsevier Applied Science, London, 1983) p. 299.

7. M. SHUBHA, MPhil dissertation, Mangalore University (1989).

8. Y. TEREMONIA and P. SMITH, Polymer 27 (1986) 1845.

Received 16 January

and accepted 24 July 1992 